\title{
EVALUATION OF THE ADM MASS AND CENTER OF MASS VIA THE RICCI TENSOR
}

\author{
PENGZI MIAO AND LUEN-FAI TAM
}

(Communicated by Lei $\mathrm{Ni}$ )

\begin{abstract}
We prove directly without using a density theorem that (i) the ADM mass defined in the usual way on an asymptotically flat manifold is equal to the mass defined intrinsically using the Ricci tensor; (ii) the Hamiltonian formulation of center of mass and the center of mass defined intrinsically using the Ricci tensor are the same.
\end{abstract}

\section{INTRODUCTION}

Let $\left(M^{n}, g\right)$ be an end of some asymptotically flat manifold, i.e. $M^{n}$ is diffeomorphic to $\mathbb{R}^{n} \backslash B(1)$, where $B(r)=\{|x|<r\}$, such that in the coordinates $\left\{x^{i}\right\}$ on $\mathbb{R}^{n}$, $g_{i j}-\delta_{i j}$ together with its derivatives decays at infinity, which will be made precise later. We will use the Einstein summation convention throughout this paper, i.e. summation over any pair of repeated indices.

For each large $r$, let

$$
\mathfrak{m}(r)=\frac{1}{2(n-1) \omega_{n-1}} \int_{S_{r}}\left(g_{i j, j}-g_{j j, i}\right) \nu_{e}^{i} d \sigma_{e},
$$

where $S_{r}=\partial B(r), \nu_{e}$ is the unit outward normal, $d \sigma_{e}$ is the area element on $S_{r}$ with respect to the Euclidean metric, and $\omega_{n-1}$ is the area of the unit sphere in $\mathbb{R}^{n}$. The ADM mass [1] of $\left(M^{n}, g\right)$ is defined as

$$
\mathfrak{m}=\lim _{r \rightarrow \infty} \mathfrak{m}(r)
$$

provided the limit exists. Under suitable conditions, it was proved by Bartnik 3 . and Chruściel [9] independently that $\mathfrak{m}$ is defined and does not depend on the choice of coordinates.

In the general relativity literature (see Ashtekar-Hansen [2] and Chruściel [10]), it is also known that the ADM mass $\mathfrak{m}$ can be computed using the curvature of $g$ as follows 1 Consider

$$
\mathfrak{m}_{I}(r)=\frac{1}{(n-1)(n-2) \omega_{n-1}} \int_{S_{r}}\left(\operatorname{Ric}-\frac{1}{2} R_{g} g\right)\left(X, \nu_{g}\right) d \sigma_{g},
$$

Received by the editors August 26, 2014 and, in revised form, January 16, 2015.

2010 Mathematics Subject Classification. Primary 83C99; Secondary 53C20.

The first author's research was partially supported by Simons Foundation Collaboration Grant for Mathematicians \#281105.

The second author's research was partially supported by Hong Kong RGC General Research Fund \#CUHK 403108.

${ }^{1}$ The authors thank Piotr Chruściel for bringing to their attention the references [2] and [10]. 
where Ric and $R_{g}$ are the Ricci tensor and the scalar curvature of $g$ respectively, $X$ is the Euclidean conformal Killing vector field $x^{i} \frac{\partial}{\partial x^{i}}, \nu_{g}$ is the unit outward normal, and $d \sigma_{g}$ is the area element on $S_{r}$ with respect to $g$. If the limit

$$
\mathfrak{m}_{I}=\lim _{r \rightarrow \infty} \mathfrak{m}_{I}(r)
$$

exists, then

$$
\mathfrak{m}=\mathfrak{m}_{I} .
$$

Formula (1.5) was also suggested by Schoen (cf. [7,11]) in connection with the generalized Pohozaev identity [16]. (For a recent application of (1.5) to the content at infinity of asymptotically flat metrics, see [5, Proposition 2.2].) It can be easily checked that (1.5) holds for metrics which are conformally flat (up to higher order) near infinity. Thus a common proof of (1.5) is to apply a density theorem from [8] or [17] to reduce the general case to metrics with harmonic asymptotics. An outline of such an approach was given in 12.

When $\mathfrak{m} \neq 0$, there exist several notions of center of mass for $(M, g)$ (cf. [11,12]). Similar to the definition of $\mathfrak{m}$, the Hamiltonian formulation of center of mass $\mathbf{c}_{\mathrm{CS}}$, proposed by Regge-Teitelboim [14] (also by Beig-Ó Murchadha [4] and CorvinoSchoen [8]), is given as follows. Let

$$
c_{\mathrm{CS}}^{\alpha}(r)=\frac{1}{2(n-1) \omega_{n-1} \mathfrak{m}} \int_{S_{r}}\left[x^{\alpha}\left(g_{i j, i}-g_{i i, j}\right) \nu_{e}^{j}-\left(g_{i \alpha} \nu_{e}^{i}-g_{i i} \nu_{e}^{\alpha}\right)\right] d \sigma_{e},
$$

where $\alpha=1, \cdots, n$. Note that $g_{i \alpha}, g_{i i}$ in the second term in the integrand can be replaced by $h_{i \alpha}, h_{i i}$ where $h_{i j}:=g_{i j}-\delta_{i j}$. Let

$$
\mathbf{c}_{\mathrm{CS}}(r)=\left(c_{\mathrm{CS}}^{1}(r), c_{\mathrm{CS}}^{2}(r), \ldots, c_{\mathrm{CS}}^{n}(r)\right) \text {; }
$$

then define

$$
\mathbf{c}_{\mathrm{CS}}=\lim _{r \rightarrow \infty} \mathbf{c}_{\mathrm{CS}}(r),
$$

provided the limit exists. (Here we use the notation $\mathbf{c}_{\mathrm{CS}}$ following [11.) Similar to (1.4), Schoen suggested an intrinsic way to define the center of mass (cf. [11]): for $\alpha=1, \ldots, n$, let $Y_{(\alpha)}$ be the Euclidean conformal Killing vector field

$$
\left(|x|^{2} \delta^{\alpha i}-2 x^{\alpha} x^{i}\right) \frac{\partial}{\partial x^{i}}
$$

define

$$
c_{I}^{\alpha}(r)=\frac{1}{2(n-1)(n-2) \omega_{n-1} \mathfrak{m}} \int_{S_{r}}\left(\operatorname{Ric}-\frac{1}{2} R_{g} g\right)\left(Y_{(\alpha)}, \nu_{g}\right) d \sigma_{g}
$$

and

$$
\mathbf{c}_{I}(r)=\left(c_{I}^{1}(r), \ldots, c_{I}^{n}(r)\right) .
$$

The intrinsic center of mass is defined as

$$
\mathbf{c}_{I}=\lim _{r \rightarrow \infty} \mathbf{c}_{I}(r),
$$

provided the limit exists. We also want to mention that Huisken-Yau [13] and Ye [18] constructed a foliation of stable constant mean curvature spheres near infinity on asymptotically Schwarzschild manifolds via different methods. Huisken-Yau 13. proposed a geometric definition of center of mass using the foliation. It was proved by Huang [11] that under the Regge-Teitelboim condition (see Theorem 1.1 (b)), all these notions of center of mass are equivalent. In order to show $\mathbf{c}_{\mathrm{CS}}=\mathbf{c}_{I}$, in [1] 
Huang first proved a density theorem for metrics satisfying the Regge-Teitelboim condition and then applied it to reduce the general case to metrics with harmonic asymptotics. Recently, in [15], Nerz also used $\mathfrak{m}_{I}$ as the definition of mass in his construction of foliations of constant mean curvature spheres for asymptotically flat manifolds under weaker asymptotic conditions.

In this paper, we give a direct proof of $\mathbf{c}_{\mathrm{CS}}=\mathbf{c}_{I}$ and $\mathfrak{m}=\mathfrak{m}_{I}$ without using density theorems. More precisely, we will prove the following:

Theorem 1.1. On an asymptotically flat end $\left(M^{n}, g\right)$ which satisfies $g_{i j}-\delta_{i j}=$ $o_{2}\left(|x|^{-\frac{n-2}{2}}\right)$, one has

(a)

$$
\lim _{r \rightarrow \infty}\left(\mathfrak{m}(r)-\mathfrak{m}_{I}(r)\right)=0 .
$$

(b) If the Regge-Teitelboim condition holds, i.e. $g_{i j}^{\text {odd }}(x)=o_{2}\left(|x|^{-\frac{n}{2}}\right)$, then

$$
\lim _{r \rightarrow \infty}\left(\mathbf{c}_{\mathrm{CS}}(r)-\mathbf{c}_{I}(r)\right)=0 .
$$

As mentioned earlier, part (a) of Theorem 1.1 is known to experts in the relativity community; (b) was first proved by Huang [11] (under slightly different decay assumptions). Our contribution is to provide more elementary and simpler proofs.

The structure of the paper is as follows. In the next section, we prove part (a) of Theorem 1.1, In Section 3, we prove part (b).

$$
\text { 2. } \mathfrak{m}=\mathfrak{m}_{I}
$$

On an asymptotically flat end $\left(M^{n}, g\right)$, as our discussion is only on behavior of $g$ near infinity, we may extend $\left(M^{n}, g\right)$ so that $M$ is diffeomorphic to $\mathbb{R}^{n}$. We will assume this throughout the rest of the paper.

Definition 1. Let $f$ be a function defined near infinity of $\mathbb{R}^{n}$. We say that $f=$ $o_{k}\left(|x|^{-\tau}\right)$, if $f$ is in $C^{k}$ and $|x|^{|\alpha|+\tau}\left|\partial^{\alpha} f(x)\right|=o(1)$ as $x \rightarrow \infty$ for all $\alpha$ with $0 \leq|\alpha| \leq k$.

Theorem 2.1. Suppose $g_{i j}-\delta_{i j}=o_{2}\left(|x|^{-\frac{n-2}{2}}\right)$ on $\left(M^{n}, g\right)$. Then

$$
\lim _{r \rightarrow \infty}\left(\mathfrak{m}(r)-\mathfrak{m}_{I}(r)\right)=0 .
$$

In fact, the following is true. Let $\left\{D_{l}\right\}_{l=1}^{\infty}$ be a sequence of bounded open sets with Lipschitz boundary $\Sigma_{l}:=\partial D_{l}$ which has area $\left|\Sigma_{l}\right|$. Let $r_{l}:=\inf _{x \in \Sigma_{l}}|x|$. Assume

$$
\lim _{l \rightarrow \infty} r_{l}=\infty \text { and }\left|\Sigma_{l}\right| \leq C r_{l}^{n-1}
$$

for some constant $C$ independent of $l$. Then

$$
\lim _{l \rightarrow \infty}\left(\mathfrak{m}(l)-\mathfrak{m}_{I}(l)\right)=0,
$$

where $\mathfrak{m}(l)$ is the RHS in (1.1) integrating over $\Sigma_{l}$ and $\mathfrak{m}_{I}(l)$ is the RHS in (1.3) integrating over $\Sigma_{l}$. 
Proof. The condition $g_{i j}-\delta_{i j}=o_{2}\left(|x|^{-\frac{n-2}{2}}\right)$ shows

$$
\left|\nu_{e}^{i}-\nu_{g}^{i}\right|=o\left(r_{l}^{-\frac{n-2}{2}}\right), \quad d \sigma_{g}=\left(1+o\left(r_{l}^{-\frac{n-2}{2}}\right)\right) d \sigma_{e}
$$

on $\Sigma_{l}$, and

$$
\begin{aligned}
2 R_{i j}(x) & =2 \frac{\partial}{\partial x^{k}} \Gamma_{j i}^{k}-2 \frac{\partial}{\partial x^{j}} \Gamma_{k i}^{k}+2 \Gamma_{k l}^{k} \Gamma_{j i}^{l}-2 \Gamma_{j l}^{k} \Gamma_{k i}^{l} \\
& =\frac{\partial}{\partial x^{k}}\left(g_{k i, j}+g_{k j, i}-g_{i j, k}\right)-\frac{\partial}{\partial x^{j}}\left(g_{k i, k}+g_{k k, i}-g_{k i, k}\right)+o\left(|x|^{-n}\right) \\
& =g_{k i, k j}+g_{k j, k i}-g_{i j, k k}-g_{k k, i j}+o\left(|x|^{-n}\right) \\
& =o\left(|x|^{-\frac{n+2}{2}}\right) .
\end{aligned}
$$

Using the fact that $\left|\Sigma_{l}\right| \leq C r_{l}^{n-1}$, by (2.2) as $l \rightarrow \infty$ we have

$$
\begin{aligned}
-2 \int_{\Sigma_{l}} R_{i j} x^{i} \nu_{g}^{j} d \sigma_{g} & =-2 \int_{\Sigma_{l}} R_{i j} x^{i} \nu_{e}^{j} d \sigma_{g}-2 \int_{\Sigma_{l}} R_{i j} x^{i}\left(\nu_{g}^{j}-\nu_{e}^{j}\right) d \sigma_{g} \\
& =-2 \int_{\Sigma_{l}} R_{i j} x^{i} \nu_{e}^{j} d \sigma_{e}+o(1) \\
& =\int_{\Sigma_{l}}\left(-g_{k i, k j}-g_{k j, k i}+g_{i j, k k}+g_{k k, i j}\right) x^{i} \nu_{e}^{j} d \sigma_{e}+o(1) .
\end{aligned}
$$

We claim that

$$
\begin{aligned}
& \int_{\Sigma_{l}}\left(-g_{k i, k j}-g_{k j, k i}+g_{i j, k k}+g_{k k, i j}\right) x^{i} \nu_{e}^{j} d \sigma_{e} \\
= & (n-2) \int_{\Sigma_{l}}\left(g_{k j, k}-g_{k k, j}\right) \nu_{e}^{j} d \sigma_{e}+\int_{\Sigma_{l}}\left(-g_{k j, k j}+g_{k k, j j}\right) x^{i} \nu_{e}^{i} d \sigma_{e} .
\end{aligned}
$$

If the claim is true, then by (2.3) and (2.4), we have

$$
\begin{aligned}
& -2 \int_{\Sigma_{l}} R_{i j} x^{i} \nu_{g}^{j} d \sigma_{g} \\
= & (n-2) \int_{\Sigma_{l}}\left(g_{k j, k}-g_{k k, j}\right) \nu_{e}^{j} d \sigma_{e}+\int_{\Sigma_{l}}\left(-g_{k j, k j}+g_{k k, j j}\right) x^{i} \nu_{e}^{i} d \sigma_{e}+o(1) .
\end{aligned}
$$

To verify (2.4), viewing each $g_{i j}$ as functions on $\mathbb{R}^{n}$, we may find sequences of smooth functions $\left\{g_{i j}^{(m)}\right\}$ such that $g_{i j}^{(m)}=g_{j i}^{(m)}$ for all $m$ and $\left\{g_{i j}^{(m)}\right\}$ converges to $g_{i j}$ uniformly in $C^{2}$ norm on any compact sets. Hence to prove (2.4), we may 
assume that $g$ is $C^{3}$. Integrating by parts on $D_{l}$ (since $\partial D_{l}$ is Lipschitz), we have

$$
\begin{aligned}
& \int_{\Sigma_{l}}\left(-g_{k i, k j}-g_{k j, k i}+g_{i j, k k}+g_{k k, i j}\right) x^{i} \nu_{e}^{j} d \sigma_{e} \\
& \int_{D_{l}} \frac{\partial}{\partial x^{j}}\left[\left(-g_{k i, k j}-g_{k j, k i}+g_{i j, k k}+g_{k k, i j}\right) x^{i}\right] d v_{e} \\
= & \int_{D_{l}}\left(-g_{k i, k j}-g_{k j, k i}+g_{i j, k k}+g_{k k, i j}\right) \delta_{j}^{i} d v_{e} \\
& +\int_{D_{l}}\left(-g_{k i, k j j}-g_{k j, k i j}+g_{i j, k k j}+g_{k k, i j j}\right) x^{i} d v_{e} \\
= & \int_{D_{l}}\left(g_{k k, j j}-g_{k j, k j}\right) d v_{e}+\int_{D_{l}}\left(-g_{k j, k i j}+g_{k k, i j j}\right) x^{i} d v_{e} \\
& \left(\operatorname{since} \sum_{k, j}\left(-g_{k i, k j j}+g_{i j, k k j}\right)=0\right) \\
= & (n-2) \int_{D_{l}}\left(g_{k j, k j}-g_{k k, j j}\right) d v_{e}+\int_{D_{l}} \frac{\partial}{\partial x^{i}}\left(\left(-g_{k j, k j}+g_{k k, j j}\right) x^{i}\right) d v_{e} \\
= & (n-2) \int_{\Sigma_{l}}\left(g_{k j, k}-g_{k k, j}\right) \nu_{e}^{j} d \sigma_{e}+\int_{\Sigma_{l}}\left(-g_{k j, k j}+g_{k k, j j}\right) x^{i} \nu_{e}^{i} d \sigma_{e},
\end{aligned}
$$

where $d v_{e}$ is the volume element with respect to the Euclidean metric. This proves (2.4).

On the other hand, by (2.2), we have

$$
\begin{aligned}
R_{g}(x) & =\sum_{i} R_{i i}+o\left(|x|^{-n}\right) \\
& =\frac{1}{2} \sum_{i, k}\left(g_{k i, k i}+g_{k i, k i}-g_{i i, k k}-g_{k k, i i}\right)+o\left(|x|^{-n}\right) \\
& =g_{i k, i k}-g_{k k, i i}+o\left(|x|^{-n}\right) \\
& =o\left(|x|^{-\frac{n+2}{2}}\right) .
\end{aligned}
$$

Hence

$$
\begin{aligned}
\int_{\Sigma_{l}}\left(-g_{k j, k j}+g_{k k, j j}\right) x^{i} \nu_{e}^{i} d \sigma_{e} & =-\int_{\Sigma_{l}} R_{g} x^{i} \nu_{e}^{i} d \sigma_{e}+o(1) \\
& =-\int_{\Sigma_{l}} R_{g} g\left(X, \nu_{g}\right) d \sigma_{g}+o(1) .
\end{aligned}
$$

Combining this with (2.5), we conclude that

$$
-2 \int_{\Sigma_{l}}\left(\operatorname{Ric}-\frac{1}{2} R_{g} g\right)\left(X, \nu_{g}\right) d \sigma_{g}=(n-2) \int_{\Sigma_{l}}\left(g_{k j, k}-g_{k k, j}\right) \nu_{e}^{j} e \sigma_{g}+o(1)
$$

as $l \rightarrow \infty$. From this it is easy to see the theorem is true.

$$
\text { 3. } \mathbf{c}_{\mathrm{CS}}=\mathbf{c}_{I}
$$

Definition 2. For a function $f(x)$ defined on $\mathbb{R}^{n}$, let

$$
f^{\text {odd }}(x):=\frac{1}{2}(f(x)-f(-x)), \quad f^{\text {even }}(x):=\frac{1}{2}(f(x)+f(-x)) .
$$


Theorem 3.1. Suppose $g_{i j}-\delta_{i j}=o_{2}\left(|x|^{-\frac{n-2}{2}}\right)$ on $\left(M^{n}, g\right)$. Suppose $g$ also satisfies the Regge-Teitelboim condition, $g_{i j}^{\text {odd }}(x)=o_{2}\left(|x|^{-\frac{n}{2}}\right)$. Then

$$
\lim _{r \rightarrow \infty}\left(\mathbf{c}_{\mathrm{CS}}(r)-\mathbf{c}_{I}(r)\right)=0
$$

Proof. For $x \in S_{r}$, we have the following:

$$
\begin{cases}g_{i j}(x)= & \left(g_{i j}\right)^{\text {even }}(x)+o\left(|x|^{-\frac{n}{2}}\right), \\ \frac{\partial g_{i j}}{\partial x^{k}}(x)= & \left(\frac{\partial g_{i j}}{\partial x^{k}}\right)^{\text {odd }}(x)+o\left(|x|^{-1-\frac{n}{2}}\right), \\ \Gamma_{i j}^{k}(x)= & \left(\Gamma_{i j}^{k}\right)^{\text {odd }}(x)+o\left(|x|^{-1-\frac{n}{2}}\right), \\ R_{i j}(x)= & \left(R_{i j}\right)^{\text {even }}(x)+o\left(|x|^{-2-\frac{n}{2}}\right), \\ R_{g}(x)= & \left(R_{g}\right)^{\text {even }}(x)+o\left(|x|^{-2-\frac{n}{2}}\right) .\end{cases}
$$

We also have

$$
\begin{aligned}
\nu_{g}^{i}(x)-\nu_{e}^{i}(x) & =\left(\nu_{g}^{i}-\nu_{e}^{i}\right)^{\text {odd }}(x)+o\left(|x|^{-\frac{n}{2}}\right), \\
f(x)-1 & =(f-1)^{\text {even }}(x)+o\left(|x|^{-\frac{n}{2}}\right),
\end{aligned}
$$

where $f(x)$ is defined by $d \sigma_{g}(x)=f(x) d \sigma_{e}(x)$. For each $\alpha=1, \ldots, n$, define $Y_{(\alpha)}(x)=Y_{(\alpha)}^{i} \frac{\partial}{\partial x^{i}}$, where $Y_{(\alpha)}^{i}=\left(|x|^{2} \delta^{\alpha i}-2 x^{\alpha} x^{i}\right)$. Note that $Y^{i}(x)=Y^{i}(-x)$. By (3.1)-(3.3), we have as $r \rightarrow \infty$,

$$
\begin{aligned}
\int_{S_{r}} \operatorname{Ric}\left(Y, \nu_{g}\right) d \sigma_{g}= & \int_{S_{r}} \operatorname{Ric}\left(Y, \nu_{g}-\nu_{e}\right)(f-1) d \sigma_{e}+\int_{S_{r}} \operatorname{Ric}\left(Y, \nu_{g}-\nu_{e}\right) d \sigma_{e} \\
& +\int_{S_{r}} \operatorname{Ric}\left(Y, \nu_{e}\right)(f-1) d \sigma_{e}+\int_{S_{r}} \operatorname{Ric}\left(Y, \nu_{e}\right) d \sigma_{e} \\
= & \int_{S_{r}} \operatorname{Ric}\left(Y, \nu_{e}\right) d \sigma_{e}+o(1),
\end{aligned}
$$

where we have also used the fact $g_{i j}(x)-\delta_{i j}=o_{2}\left(|x|^{-\frac{n-2}{2}}\right)$. Using (3.1) and (2.2), as $r \rightarrow \infty$, we then have

$$
\begin{aligned}
& 2 \int_{S_{r}} R_{i j} Y^{i} \nu_{e}^{j} d \sigma_{e} \\
= & 2 \int_{S_{r}}\left(\frac{\partial}{\partial x^{k}} \Gamma_{j i}^{k}-\frac{\partial}{\partial x^{j}} \Gamma_{k i}^{k}\right) Y^{i} \nu_{e}^{j} d \sigma_{e}+o(1) \\
= & \int_{S_{r}}\left[\left(g^{k s}\left(g_{i s, j k}+g_{j s, i k}-g_{i j, s k}\right)-g^{k s}\left(g_{k s, i j}+g_{i s, k j}-g_{k i, s j}\right)\right] Y^{i} \nu_{e}^{j} d \sigma_{e}+o(1)\right. \\
= & \int_{S_{r}}\left(g_{i k, j k}+g_{j k, i k}-g_{i j, k k}-g_{k k, i j}\right) Y^{i} \nu_{e}^{j} d \sigma_{e}+o(1) .
\end{aligned}
$$


As in the proof of Theorem 2.1, we may assume that $g$ is smooth to obtain

$$
\begin{aligned}
& \int_{S_{r}}\left(g_{k i, k j}+g_{k j, k i}-g_{i j, k k}-g_{k k, i j}\right) Y^{i} \nu_{e}^{j} d \sigma_{e} \\
= & \int_{B(r)} \frac{\partial}{\partial x^{j}}\left[\left(g_{k i, k j}+g_{k j, k i}-g_{i j, k k}-g_{k k, i j}\right) Y^{i}\right] d v_{e} \\
= & \int_{B(r)}\left(g_{k j, k i j}-g_{k k, i j j}\right) Y^{i} d v_{e}+\int_{B(r)}\left(g_{k i, k j}+g_{k j, k i}-g_{i j, k k}-g_{k k, i j}\right) \frac{\partial}{\partial x^{j}} Y^{i} d v_{e} \\
= & \int_{S_{r}}\left(g_{k j, k j}-g_{k k, j j}\right) Y^{i} \nu_{e}^{i} d \sigma_{e}-\int_{B(r)}\left(g_{k j, k j}-g_{k k, j j}\right) \frac{\partial}{\partial x^{i}} Y^{i} d v_{e} \\
& +\int_{B(r)}\left(g_{k i, k j}+g_{k j, k i}-g_{i j, k k}-g_{k k, i j}\right) \frac{\partial}{\partial x^{j}} Y^{i} d v_{e} .
\end{aligned}
$$

Since

$$
\frac{\partial}{\partial x^{j}} Y^{i}=\frac{\partial}{\partial x^{j}}\left(|x|^{2} \delta^{\alpha i}-2 x^{\alpha} x^{i}\right)=2 x^{j} \delta^{\alpha i}-2 \delta^{\alpha j} x^{i}-2 x^{\alpha} \delta_{j}^{i},
$$

we have

$$
\left(g_{k j, k j}-g_{k k, j j}\right) \frac{\partial}{\partial x^{i}} Y^{i}=-2 n x^{\alpha}\left(g_{k j, k j}-g_{k k, j j}\right),
$$

and

$$
\begin{aligned}
\left(g_{k i, k j}+g_{k j, k i}-g_{i j, k k}-g_{k k, i j}\right) \frac{\partial}{\partial x^{j}} Y^{i} & =-2 x^{\alpha}\left(g_{k i, k i}+g_{k i, k i}-g_{i i, k k}-g_{k k, i i}\right) \\
& =-4 x^{\alpha}\left(g_{k i, k i}-g_{k k, i i}\right) .
\end{aligned}
$$

Hence

$$
\begin{aligned}
& \int_{S_{r}}\left(g_{k i, k j}+g_{k j, k i}-g_{i j, k k}-g_{k k, i j}\right) Y^{i} \nu_{e}^{j} d \sigma_{e} \\
= & \int_{S_{r}}\left(g_{k j, k j}-g_{k k, j j}\right) Y^{i} \nu_{e}^{j} d \sigma_{e}+2(n-2) \int_{B(r)} x^{\alpha}\left(g_{k i, k i}-g_{k k, i i}\right) d v_{e} \\
= & \int_{S_{r}}\left(g_{k j, k j}-g_{k k, j j}\right) Y^{i} \nu_{e}^{i} d \sigma_{e}+2(n-2) \int_{S_{r}} x^{\alpha}\left(g_{k i, k}-g_{k k, i}\right) \nu_{e}^{i} d \sigma_{e} \\
& -2(n-2) \int_{B(r)}\left(g_{k \alpha, k}-g_{k k, \alpha}\right) d v_{e} \\
= & \int_{S_{r}}\left(g_{k j, k j}-g_{k k, j j}\right) Y^{i} \nu_{e}^{i} d \sigma_{e} \\
& +2(n-2) \int_{S_{r}}\left[x^{\alpha}\left(g_{k i, k}-g_{k k, i}\right) \nu_{e}^{i}-\left(g_{k \alpha} \nu_{e}^{k}-g_{k k} \nu_{e}^{\alpha}\right)\right] d \sigma_{e} .
\end{aligned}
$$

Here $g_{k \alpha}, g_{k k}$ in the second term in the integrand of the second integral in the above can be replaced by $h_{k \alpha}, h_{k k}$ where $h_{i j}:=g_{i j}-\delta_{i j}$. 
Using (3.1)-(3.3), we may argue as before to conclude that

$$
\int_{S_{r}}\left(g_{k j, k j}-g_{k k, j j}\right) Y^{i} \nu_{e}^{i} d \sigma_{e}=\int_{S_{r}} R_{g} g\left(Y, \nu_{g}\right) d \sigma_{g}+o(1)
$$

and

$$
\begin{aligned}
& \int_{S_{r}}\left[x^{\alpha}\left(g_{k i, k}-g_{k k, i}\right) \nu_{e}^{i}-\left(g_{k \alpha} \nu_{e}^{k}-g_{k k} \nu_{e}^{\alpha}\right)\right] d \sigma_{e} \\
& =\int_{S_{r}}\left[x^{\alpha}\left(g_{k i, k}-g_{k k, i}\right) \nu_{g}^{i}-\left(g_{k \alpha} \nu_{g}^{k}-g_{k k} \nu_{g}^{\alpha}\right)\right] d \sigma_{g}+o(1),
\end{aligned}
$$

as $r \rightarrow \infty$. Combining these with (3.4) and (3.5), and using (2.6) for the expression of $R_{g}$, we have

$$
\begin{aligned}
& \int_{S_{r}}\left(R_{i j}-\frac{1}{2} R_{g} g_{i j}\right) Y^{i} \nu_{g}^{j} d \sigma_{g} \\
& =(n-2) \int_{S_{r}}\left[x^{\alpha}\left(g_{k i, k}-g_{k k, i}\right) \nu_{e}^{i} d \sigma_{e}-\left(g_{k \alpha} \nu_{e}^{k}-g_{k k} \nu^{\alpha}\right)\right] d \sigma_{e}+o(1) \\
& =(n-2) \int_{S_{r}}\left[x^{\alpha}\left(g_{k i, k}-g_{k k, i}\right) \nu_{g}^{i}-\left(g_{k \alpha} \nu_{g}^{k}-g_{k k} \nu_{g}^{\alpha}\right)\right] d \sigma_{g}+o(1),
\end{aligned}
$$

as $r \rightarrow \infty$. From this the result follows.

Remark 3.1. On an asymptotically flat $\left(M^{n}, g\right)$ with $g_{i j}-\delta_{i j}=O_{2}\left(|x|^{-q}\right)$, where $q>\frac{n-2}{2}$, it follows from the proof of Theorem 2.1 in Section 2 that $\mathfrak{m}$ and hence $\mathfrak{m}_{I}$ are defined if and only if $\lim _{r \rightarrow \infty} \int_{B(r)} R_{g} d v_{g}$ exists. On the other hand, if in addition $g^{\text {odd }}=O_{2}\left(|x|^{-q-1}\right.$ ), the computation in [8] (also cf. [6]) shows $\mathbf{c}_{\mathrm{CS}}$ and hence $\mathbf{c}_{I}$ are defined if and only if $\lim _{r \rightarrow \infty} \int_{B(r)} x^{i} R_{g} d v_{g}$ exists for $i=1, \ldots, n$. Here $f=O_{2}\left(|x|^{-q}\right)$ means $|x|^{|\alpha|+q}\left|\partial^{\alpha} f\right| \leq C$ for all $\alpha$ with $|\alpha| \leq 2$.

\section{ACKNOWLEDGEMENT}

The authors would like to thank Richard Schoen for useful discussions.

\section{REFERENCES}

[1] Richard Arnowitt, Stanley Deser, and Charles W. Misner, Coordinate invariance and energy expressions in general relativity., Phys. Rev. (2) 122 (1961), 997-1006. MR0127946 (23 \#B991)

[2] Abhay Ashtekar and R. O. Hansen, A unified treatment of null and spatial infinity in general relativity. I. Universal structure, asymptotic symmetries, and conserved quantities at spatial infinity, J. Math. Phys. 19 (1978), no. 7, 1542-1566. MR0503432 (58 \#20188)

[3] Robert Bartnik, The mass of an asymptotically flat manifold, Comm. Pure Appl. Math. 39 (1986), no. 5, 661-693, DOI 10.1002/cpa.3160390505. MR849427 (88b:58144)

[4] Robert Beig and Niall Ó Murchadha, The Poincaré group as the symmetry group of canonical general relativity, Ann. Physics 174 (1987), no. 2, 463-498, DOI 10.1016/0003-4916(87)900376. MR881482(88c:83046)

[5] Alessandro Carlotto and Richard M. Schoen, Localizing solutions of the Einstein constraint equations, arXiv: 1407.4766.

[6] Pak Yeung Chan and Luen-Fai Tam, A note on center of mass, arXiv:1402.1220, to appear in Comm. Anal. Geom.

[7] Justin Corvino and Daniel Pollack, Scalar curvature and the Einstein constraint equations, Surveys in geometric analysis and relativity, Adv. Lect. Math. (ALM), vol. 20, Int. Press, Somerville, MA, 2011, pp. 145-188. MR2906924 
[8] Justin Corvino and Richard M. Schoen, On the asymptotics for the vacuum Einstein constraint equations, J. Differential Geom. 73 (2006), no. 2, 185-217. MR2225517(2007e:58044)

[9] Piotr T. Chruściel, Boundary conditions at spatial infinity from a Hamiltonian point of view, Topological properties and global structure of space-time (Erice, 1985), NATO Adv. Sci. Inst. Ser. B Phys., vol. 138, Plenum, New York, 1986, pp. 49-59. MR.1102938 (92h:83003)

[10] Piotr T. Chruściel, A remark on the positive-energy theorem, Classical Quantum Gravity 3 (1986), no. 6, L115-L121. MR868711 (88f:83030a)

[11] Lan-Hsuan Huang, On the center of mass of isolated systems with general asymptotics, Classical Quantum Gravity 26 (2009), no. 1, 015012, 25, DOI 10.1088/0264-9381/26/1/015012. MR2470255(2010e:83009)

[12] Lan-Hsuan Huang, On the center of mass in general relativity, Fifth International Congress of Chinese Mathematicians. Part 1, 2, AMS/IP Stud. Adv. Math., 51, pt. 1, vol. 2, Amer. Math. Soc., Providence, RI, 2012, pp. 575-591. MR2908093

[13] Gerhard Huisken and Shing-Tung Yau, Definition of center of mass for isolated physical systems and unique foliations by stable spheres with constant mean curvature, Invent. Math. 124 (1996), no. 1-3, 281-311, DOI 10.1007/s002220050054. MR.1369419 (96m:53037)

[14] Tullio Regge and Claudio Teitelboim, Role of surface integrals in the Hamiltonian formulation of general relativity, Ann. Physics 88 (1974), 286-318. MR0359663(50 \#12115)

[15] Christopher Nerz, Foliations by stable spheres with constant mean curvature for isolated systems without asymptotic symmetry, arXiv:1408.0752.

[16] Richard M. Schoen, The existence of weak solutions with prescribed singular behavior for a conformally invariant scalar equation, Comm. Pure Appl. Math. 41 (1988), no. 3, 317-392, DOI 10.1002/cpa.3160410305. MR.929283 (89e:58119)

[17] Richard M. Schoen and Shing Tung Yau, The energy and the linear momentum of space-times in general relativity, Comm. Math. Phys. 79 (1981), no. 1, 47-51. MR609227 (82j:83045)

[18] Rugang Ye, Foliation by constant mean curvature spheres on asymptotically flat manifolds, Geometric analysis and the calculus of variations, Int. Press, Cambridge, MA, 1996, pp. 369383. MR 1449417 (98e:53040)

Department of Mathematics, University of Miami, Coral Gables, Florida 33146

E-mail address: pengzim@math.miami.edu

The Institute of Mathematical Sciences and Department of Mathematics, The Chinese University of Hong Kong, Shatin, Hong Kong, People's Republic of China

E-mail address: Iftam@math.cuhk.edu.hk 